\title{
Avoiding the Falciform Ligament Sign during the Intraoperative Cholangiogram
}

\author{
Saksham Gupta ${ }^{1}$, Simon Whitcher ${ }^{2}$
}

\begin{abstract}
We have observed that the falciform ligament can appear prominently as a vertical lucent artifact making cholangiography difficult during laparoscopic cholecystectomy. Our suspicion is that this is due to the pneumoperitoneum, and once the pneumoperitoneum is released, this artifact disappears. We have presented images displaying this phenomenon that we feel would be useful for general surgeons operating on the gallbladder.

Keywords: Cholangiography, Falciform ligament, Laparoscopic cholecystectomy.

World Journal of Laparoscopic Surgery (2021): 10.5005/jp-journals-10033-1461
\end{abstract}

With the advent of laparoscopic cholecystectomy as the choice for surgery on the gallbladder, the intraoperative cholangiogram has been an adjunct to assess the biliary anatomy to reduce the risk of bile duct injuries. ${ }^{1}$ Furthermore, this cholangiogram allows the operating surgeon to evaluate for filling defects within the distal bile duct and confirm flow into the duodenum and becomes necessary for any common bile duct exploration. The surgeon needs to be equipped with strategies on how to achieve the best cholangiography images. We would like to report a simple technique on improving intraoperative cholangiography images during laparoscopic cholecystectomy, which to our knowledge has not yet been described.

The "falciform ligament sign" has been described as a vertical lucent artifact in situations where the patient has a pneumoperitoneum on a plain abdominal radiograph in the setting of hollow viscous perforation. ${ }^{2,3}$ With pneumoperitoneum, the falciform ligament becomes taut and lies in the axis of the sagittal plane and in this orientation, would potentially obscure
1,2 John Hunter Hospital, New Lambton Heights, New South Wales, Australia

Corresponding Author: Saksham Gupta, John Hunter Hospital, New Lambton Heights, New South Wales, Australia, Phone: +61249213000, e-mail: saksham_gupta@live.com.au

How to cite this article: Gupta S, Whitcher S. Avoiding the Falciform Ligament Sign during the Intraoperative Cholangiogram. World J Lap Surg 2021;14(2):136-137.

Source of support: Nil

Conflict of interest: None

more of the X-ray beam. We have observed a similar sign during the artificial creation of pneumoperitoneum during laparoscopic cholecystectomy. Surgeons need to be weary not to mistake this as a contrast leak or a biliary structure during the intraoperative cholangiogram. Even if this sign is correctly recognized, it can
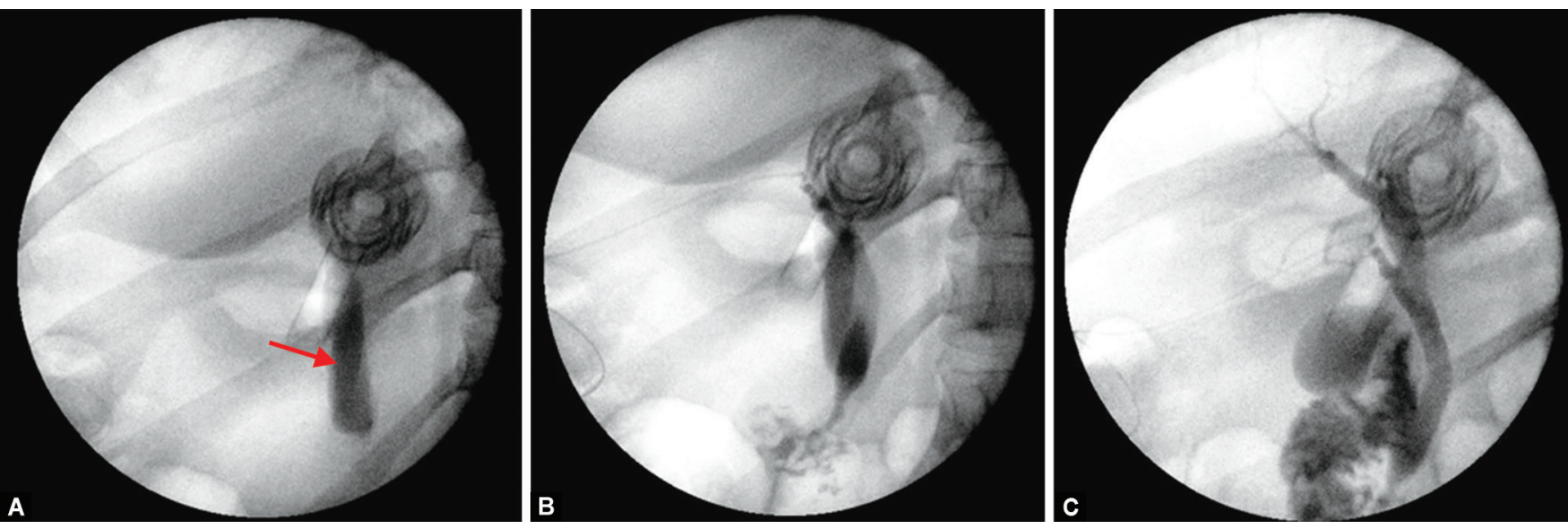

Figs $1 \mathrm{~A}$ to C: Sequential cholangiogram images taken during an elective laparoscopic cholecystectomy. (A) Scout film taken before the introduction of contrast dye through the cholangiogram catheter. A radiolucent artifact (red arrow) is seen below the epigastric port. This is the falciform ligament sign; (B) Attempt made to achieve cholangiogram image; however, this artifact completely overlies the common bile duct. (C) On removal of the pneumoperitoneum, the shadow disappears, and the common bile duct is better visualized, including the entry of the cystic duct.

() Jaypee Brothers Medical Publishers. 2021 Open Access This article is distributed under the terms of the Creative Commons Attribution 4.0 International License (https://creativecommons.org/licenses/by-nc/4.0/), which permits unrestricted use, distribution, and non-commercial reproduction in any medium, provided you give appropriate credit to the original author(s) and the source, provide a link to the Creative Commons license, and indicate if changes were made. The Creative Commons Public Domain Dedication waiver (http://creativecommons.org/publicdomain/zero/1.0/) applies to the data made available in this article, unless otherwise stated. 
obscure the bile ducts making cholangiogram interpretation difficult (Figs 1A and 1B). It has been our practice that if this sign is observed, then the pneumoperitoneum can easily be released for the purpose of the cholangiogram, without any intraoperative complications. This removes the falciform ligament sign from the images, allowing clear cholangiogram images to be seen (Fig. 1C). We feel this is a simple technique that all surgeons could include in their practice during laparoscopic cholecystectomy.

\section{References}

1. Flum DR, Dellinger EP, Cheadle A, et al. Intraoperative cholangiography and risk of common bile duct injury during cholecystectomy. JAMA 2003;289:1639-1644. DOI: 10.1001/jama.289.13.1639.

2. Chou P-C, Su Y-J. Falciform ligament sign. N Engl J Med 2017;377:e28. DOI: 10.1056/NEJMicm1613914.

3. Lin J-N. Falciform-ligament sign of pneumoperitoneum. N Engl J Med 2004;351:e16. DOI: 10.1056/ENEJMicm030659. 